\title{
CCAAT/enhancer binding protein homologous protein knockdown alleviates hypoxia-induced myocardial injury in rat cardiomyocytes exposed to high glucose
}

\author{
WENQI YANG, FANG WU, TING LUO and YUELAN ZHANG
}

Department of Cardiology, The First Affiliated Hospital of China Medical University, Shenyang, Liaoning 110001, P.R. China

Received September 13, 2017; Accepted January 16, 2018

DOI: $10.3892 /$ etm.2018.5944

\begin{abstract}
Diabetic patients are more sensitive to ischemic injury than non-diabetics. Endoplasmic reticulum (ER) stress has been reported to be closely associated with the pathophysiology of ischemic injury in diabetes. The aim of the present study was to investigate the mechanisms involved in the progression of diabetes complicated by myocardial infarction (MI) and further verify the role of CCAAT/enhancer binding protein (C/EBP)-homologous protein (CHOP) using an in vitro model of diabetes/MI. The rats were exposed to $65 \mathrm{mg} / \mathrm{kg}$ streptozotocin (STZ) and left anterior descending (LAD) coronary artery ligation. ST-segment elevation, heart rate, left ventricular systolic pressure (LVSP) and LV end-diastolic pressure (LVEDP) were measured. Serum creatinine kinase-MB (CK-MB) and cardiac troponin T (cTnT) levels were examined by ELISA. Infarct size and apoptosis were measured by triphenyltetrazolium chloride staining and terminal deoxynucleotidyl-transferase-mediated dUTP nick end labeling assay. Pathological changes were evaluated by hematoxylin and eosin staining. H9c2 cells were used to establish an in vitro model of diabetes complicated by MI. Following CHOP knockdown, cell viability, cell cycle distribution and apoptosis were examined by Cell Counting Kit-8 assay, flow cytometry and Hoechst staining. Glucose-regulated protein 78 (GRP78), CHOP, B-cell lymphoma 2 (Bcl-2), Bcl-2-associated $\mathrm{X}$ protein (Bax), endoplasmic reticulum oxidoreductase 1 (Ero1)- $\alpha$, Ero1 $\beta$ and protein disulfide isomerase (PDI) levels in both myocardial tissues and $\mathrm{H} 9 \mathrm{c} 2$ cells were determined by western blotting. In the present study, diabetes complicated by MI promoted ST-segment elevation and myocardial apoptosis, increased infarct size, induced pathological changes
\end{abstract}

Correspondence to: Professor Yuelan Zhang, Department of Cardiology, The First Affiliated Hospital of China Medical University, 155 North Nanjing Street, Shenyang, Liaoning 110001, P.R. China

E-mail: zhangyuelan33@163.com

Key words: endoplasmic reticulum stress, myocardial infarction, apoptosis, CCAAT/enhancer binding protein-homologous protein, diabetes and elevated LVEDP, CK-MB, cTnT, GRP78, CHOP, Bax, Erol $\alpha$, Ero1 $\beta$ and PDI; however, it decreased heart rate, LVSP and Bcl-2. Additionally, high glucose combined with hypoxic treatment reduced cell viability, induced cell cycle arrest at G1 phase, promoted cell apoptosis, and activated the GRP78/CHOP and Ero1/PDI signaling pathways, which were reversed by CHOP knockdown. Thus, CHOP may be an effective therapeutic target for the treatment of diabetes complicated by MI.

\section{Introduction}

Cardiovascular disease (CVD) is a class of diseases that affect the blood vessels or heart (1). CVD causes 17.3 million death every year worldwide and the death is expected to increase to 23.6 million by the year 2030 (2). CVDs, including diabetic cardiomyopathy, atherosclerosis, myocardial infarction (MI) and heart failure, are the leading cause of death in diabetes (3). CVD is commonly diagnosed among Type 2 diabetics; however, the death risk of CVD in Type 1 diabetics is higher (4). Although the death rate of CVD has been decreased by $40 \%$ in U.S. diabetic adults (5), the burdens of patients remain high and effective therapeutic strategies are still lacking.

The endoplasmic reticulum (ER), the largest membrane-bound organelle in eukaryotic cells, plays important roles in protein synthesis, posttranslational modification and processing, and folding, assembly and trafficking of secretory protein and membrane (6,7). Excessive unfolded proteins lead to occurrence of ER stress and then activate a protective signaling pathway termed the unfolded protein response (UPR). The activation of UPR causes reduction of protein synthesis, promotion of correct protein folding, and degradation of misfolded proteins $(8,9)$. It has been reported that ER stress triggers apoptosis of $\beta$ cells and results in the onset of diabetes (10). Additionally, ER stress and myocardial apoptosis are associated with acute MI (AMI) development (11). Activation of CCAAT/enhancer binding protein (C/EBP)-homologous protein (CHOP) (a marker of ER stress) plays an essential role in ER stress-mediated apoptosis (12). Takada et al (13) have found that ER stress is a leading cause of left ventricular diastolic dysfunction in Type 2 diabetes. In addition, Barr et al (14) have found that ER stress is suppressed by hydrogen sulfide during treatment of cardiovascular complications in diabetes. However, the 
roles of ER stress and CHOP-induced myocardial injury in the progression of MI in diabetes have not yet been fully explored.

In the present study, whether ER stress contributed to the development of MI in diabetes in vivo was examined. The effects of CHOP knockdown on cell viability, cell cycle progression, apoptosis, ER stress and ER redox homeostasis were then investigated in rat cardiomyocytes in an in vitro model of diabetes/MI.

\section{Materials and methods}

Induction of diabetes and MI. All procedures were performed in accordance with the Guide for the Care and Use of Laboratory Animals and approved by the Institutional Animal Care and Use Committee of China Medical University (Shenyang, China). The 8-week-old Sprague-Dawley rats (Vital River Laboratories, Beijing, China) were fed with food and water ad libitum (12-h light-dark cycle) and randomly divided into 5 groups: i) Control group, ii) Diabetes group, iii) Diabetes/Sham group, iv) MI group and v) Diabetes/MI group ( $\mathrm{n}=12$ rats in each group). At $12 \mathrm{~h}$ prior to animal experiments, the rats were allowed access only to water. Diabetes was induced by $65 \mathrm{mg} / \mathrm{kg}$ streptozotocin (STZ; in $0.01 \mathrm{M}$ sodium citrate buffer, pH 4.4) (Beijing Solarbio Science \& Technology Co., Ltd., Beijing, China) via intraperitoneal injection. One week after induction of diabetes, blood glucose levels were measured and rats with glucose levels greater than $396 \mathrm{mg} / \mathrm{dl}$ were included in this study. The control rats received an injection of $0.01 \mathrm{M}$ sodium citrate buffer. MI was established by ligation of left anterior descending (LAD) coronary artery. Briefly, after measurement of blood glucose levels, anesthetization was performed and the rats were placed in the supine position. The skin was cut off using surgical scissors and the rats were ventilated with $\mathrm{HX}-300 \mathrm{~S}$ animal respirator (Chengdu Techman Software Co., Ltd., Chengdu, China; tidal volume, $6-8 \mathrm{ml} / \mathrm{kg}$; rate, 80 breaths $/ \mathrm{min})$. Then, left thoracotomy was performed and the LAD coronary artery (3-5 $\mathrm{mm}$ from the root of aorta) was ligated using nylon suture for 1 week. The rats in the Diabetes/Sham group were underwent left thoracotomy without LAD coronary artery ligation after diabetes induction. After that, the rats were anesthetized and hemodynamic parameters were measured. Then, the blood was collected and the heart was removed. The rats were euthanized by exsanguinations before tissue collection.

Electrocardiogram (ECG) and hemodynamic parameters. ECG, heart rate, left ventricular systolic pressure (LVSP) and LV end-diastolic pressure (LVEDP) were measured by Biological Data Acquisition and Analysis System BL-420 (Chengdu Techman Software Co., Ltd.).

ELISA. Serum cardiac enzymes cardiac troponin T (cTnT) and creatine kinase-MB isoenzyme (CK-MB) were measured by ELISA kits according to the manufacturer's instructions. CK-MB assay kit and ELISA kit for cTnT were purchased from WHB Scientific (Shanghai, China).

Triphenyltetrazolium chloride (TTC) staining. Infarct size was evaluated by TTC staining. After euthanasia, the heart was excised, incubated with $10 \% \mathrm{KCl}$ solution and frozen at $-20^{\circ} \mathrm{C}$. The heart was cut into slices and stained with $1 \%$ TTC solution (Beijing Solarbio Science \& Technology Co., Ltd.) at $37^{\circ} \mathrm{C}$. These slices stained with TTC were photographed. The infarct area (white) and total area (white and red) were analyzed by Image-Pro Plus software 6.0 (Media Cybernetics, Inc., Rockville, MD, USA). Infarct size (\%) was expressed as a percentage of infarct area over total area.

$H \& E$ staining. The fixed heart tissues were dehydrated in ethanol and embedded in paraffin. Then, the obtained $5-\mu$ m-thick sections were dewaxed, rehydrated and stained with hematoxylin and eosin dye. The pathological changes of H\&E-stained sections were observed under a microscope (Olympus, Tokyo, Japan; x400 magnification).

TUNEL assay. Cell apoptosis was examined by in situ Cell Death Detection kit (Roche, Mannheim, Germany) according to the manufacturer's instructions. Briefly, the fixed tissues were embedded in paraffin and sectioned into 5- $\mu$ m-thick sections. Immediately, the sections were dewaxed in xylene, rehydrated in different concentrations of ethanol and permeabilized with $0.1 \%$ Triton $\mathrm{X}-100$ at room temperature for $8 \mathrm{~min}$. After being washed with $\mathrm{PBS}$, the sections were blocked with $3 \% \mathrm{H}_{2} \mathrm{O}_{2}$ solution and incubated with TUNEL reaction mixture (Roche) at $37^{\circ} \mathrm{C}$ for $1 \mathrm{~h}$ in the dark prior to being counterstained with hematoxylin (Solarbio). The stained sections were observed under an Olympus microscope (Olympus; x400 magnification).

Western blotting. Total proteins isolated from the heart and cardiomyocytes were quantified by bicinchoninic acid (BCA) (Beyotime Institute of Biotechnology, Haimen, China) and subjected to Western blotting. Briefly, equal amounts of proteins were boiled for $5 \mathrm{~min}$ with loading buffer and then separated by SDS-PAGE. The proteins were transferred to PVDF membranes (EMD Millipore, Bedford, MA, USA) and blocked with buffer (5\% non-fat milk in TBST buffer) for $1 \mathrm{~h}$. The PVDF membranes were incubated with primary antibody against glucose-regulated protein 78 (GRP78) (D151791; Sangon Biotech, Shanghai, China; 1:500 dilution), CHOP (bs-20669R; Bioss, Beijing, China; 1:500 dilution), B-cell lymphoma 2 (Bcl-2; BA0412; Boster, Wuhan, China; 1:400 dilution), Bcl-2-associated X protein (Bax; D120073; Sangon Biotech; 1:500 dilution), endoplasmic reticulum oxidoreductase $1 \alpha$ (Erol $\alpha$; bs-10551M; Bioss; 1:500 dilution), Ero1 $\beta$ (bs-14627R; Bioss; 1:500 dilution) or protein disulfide isomerase (PDI; bs-4250R; Bioss; 1:500 dilution). After being washed with TBST buffer, the membranes were incubated with corresponding secondary antibody (A0208 and A0216; Beyotime Institute of Biotechnology; 1:5,000 dilution) at $37^{\circ} \mathrm{C}$. After being washed, the bands were developed using ECL regent (Beyotime Institute of Biotechnology) and quantified by Media Cybernetics Gel-Pro analyzer. $\beta$-actin was used as an internal control.

Grouping and treatments. Rat cardiomyocyte H9c2 cells were cultured in DMEM with $10 \% \mathrm{FBS}$ at $37^{\circ} \mathrm{C}$ and divided into 6 groups: i) Control group, normal H9c2 cells were cultured for $96 \mathrm{~h}$; ii) High glucose (HG) group, the cells were firstly maintained for $24 \mathrm{~h}$ under normal conditions and then incubated for $72 \mathrm{~h}$ with DMEM containing $33 \mathrm{mM}$ glucose before 

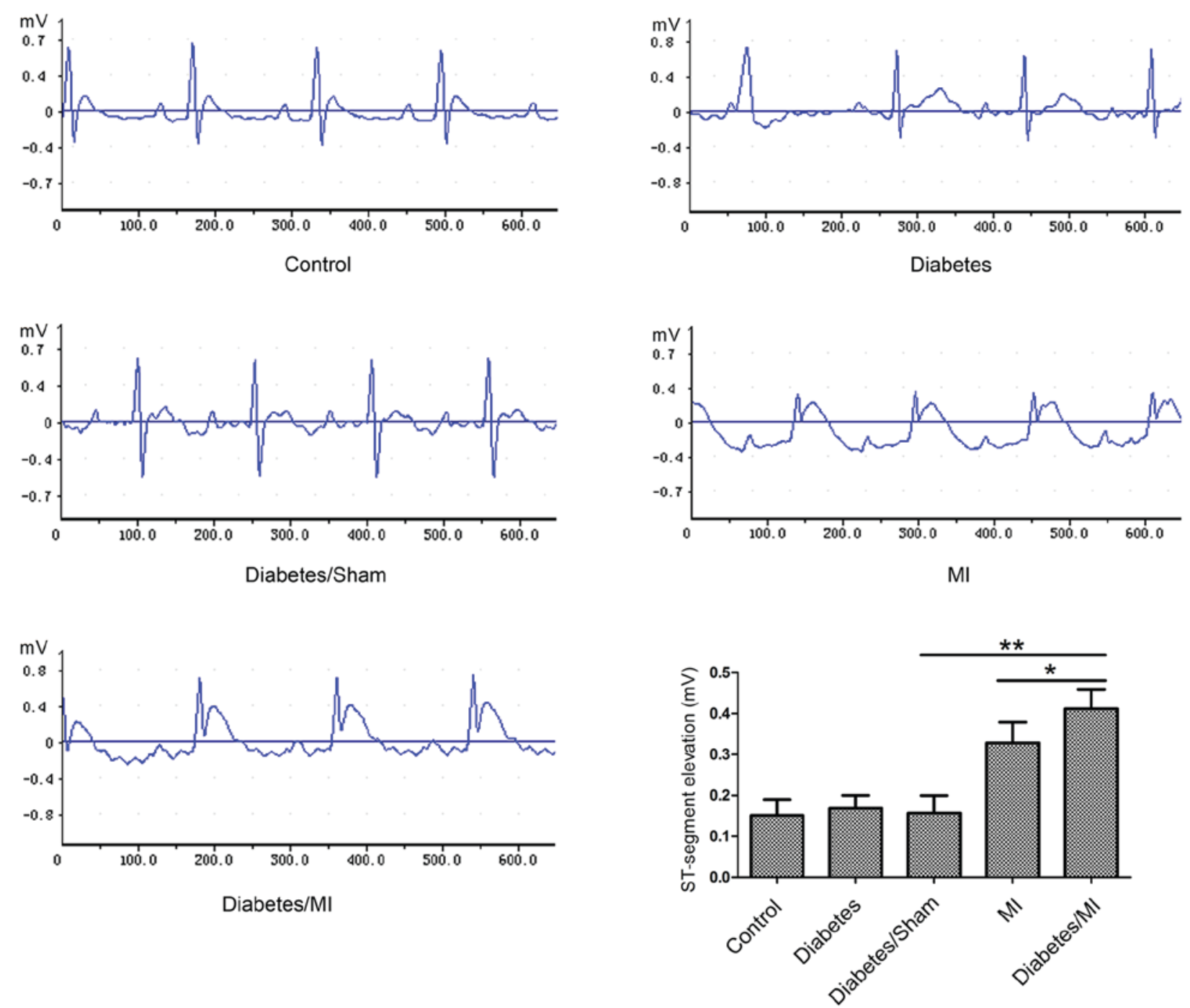

Figure 1. Electrocardiograms were recorded using a BL-420 System and the ST-segment elevation was recorded. Values are presented as the mean \pm standard deviation. ${ }^{*} \mathrm{P}<0.05$ and ${ }^{* * *} \mathrm{P}<0.01$, as indicated. MI, myocardial infarction.

analysis; iii) Hypoxia group, the cells were firstly maintained for $72 \mathrm{~h}$ under normal conditions and then cultured under a hypoxic environment $\left(3 \% \mathrm{O}_{2}, 5 \% \mathrm{CO}_{2}\right.$ and $\left.92 \% \mathrm{~N}_{2}\right)$ for $24 \mathrm{~h}$ prior to analysis; iv) $\mathrm{HG} / \mathrm{Hyp}$ pxia group, after $24 \mathrm{~h}$ of normal culture, the cells were firstly subjected to HG/Normoxia treatment for $48 \mathrm{~h}$ and then $\mathrm{HG} /$ Hypoxia treatment for $24 \mathrm{~h}$ prior to analysis; v) HG/Hypoxia/siNC group, $24 \mathrm{~h}$ after $30 \mathrm{pmol}$ siRNA (GenePharma, Shanghai, China) transfection using Lipofectamine RNAiMAX (Invitrogen; Thermo Fisher Scientific, Inc., Waltham, MA, USA) according to the manufacturer's protocol, the cells underwent $48 \mathrm{~h}$ of $\mathrm{HG} /$ Normoxia and $24 \mathrm{~h}$ of $\mathrm{HG} / \mathrm{Hypoxia}$ treatments; vi) HG/Hypoxia/siCHOP group, $24 \mathrm{~h}$ post-transfection, siCHOP-transfected H9c2 cells were subjected to $48 \mathrm{~h}$ of HG/Normoxia and $24 \mathrm{~h}$ of HG/Hypoxia treatments. Western blotting was performed to confirm the successful knockdown of CHOP expression. The sequences of siRNAs were shown below: siCHOP, 5'-GAA CUAGGAAACGGAAACATT-3'; siNC, 5'-UUCUCCGAA CGUGUCACGUTT-3'.

Cell Counting Kit-8 (CCK-8) assay. H9c2 cells were seeded in 96-well plates (at a density of 3,000 cells/well) and cultured in a
$5 \% \mathrm{CO}_{2}$ incubator. Cell viability was examined by CCK-8 assay. The cells were treated with $10 \mu \mathrm{l}$ CCK- 8 solution (Beyotime) at $37^{\circ} \mathrm{C}$ for $1 \mathrm{~h}$. The absorbance at $450 \mathrm{~nm}$ was measured by a microplate reader (BioTek, Winooski, Vermont, USA).

Cell cycle and apoptosis analysis. For cell cycle analysis, H9c2 cells were washed with PBS and fixed in $70 \%$ ethanol. The cells were incubated with $25 \mu \mathrm{l}$ propidium iodide (PI)/10 $\mu \mathrm{l}$ RNaseA in staining buffer (Beyotime Institute of Biotechnology) at $37^{\circ} \mathrm{C}$ for $30 \mathrm{~min}$ in the dark. After incubation, the cells were subjected to cytometric analysis. For apoptosis detection, the cells were treated with $5 \mu \mathrm{l}$ Annexin V-FITC/5 $\mu \mathrm{l}$ propidium iodide (PI) in binding buffer (KeyGen, Nanjing, China) for $15 \mathrm{~min}$ before analysis.

Hoechst 33258 staining. H9c2 cells were seeded onto coverslips in 12 -well plates at the density of $4 \times 10^{4}$ cells/coverslip. After the aforementioned treatment, the cells were fixed with $4 \%$ paraformaldehyde at room temperature for $20 \mathrm{~min}$ and stained with Hoechst 33258 (Beyotime Institute of Biotechnology) for 5 min. Subsequently, images were captured by a fluorescent microscope (IX53; Olympus; x400 magnification). 
Table I. Heart function.

\begin{tabular}{lccc}
\hline Group & LVEDP $(\mathrm{mm} \mathrm{Hg})$ & LVSP $(\mathrm{mm} \mathrm{Hg})$ & Heart rate (beats/min) \\
\hline Control & $5.133 \pm 0.819$ & $110.000 \pm 8.690$ & $400.167 \pm 15.651$ \\
Diabetes & $6.750 \pm 1.048$ & $99.650 \pm 9.764$ & $361.167 \pm 15.562^{\mathrm{a}}$ \\
Diabetes/Sham & $6.217 \pm 0.662$ & $95.217 \pm 9.251$ & $368.667 \pm 17.397$ \\
MI & $8.567 \pm 0.792$ & $86.300 \pm 6.165$ & $376.667 \pm 16.318$ \\
Diabetes/MI & $10.433 \pm 2.455^{\mathrm{b}}$ & $81.317 \pm 8.259$ & $321.000 \pm 15.388^{\mathrm{b}, \mathrm{c}}$ \\
\hline
\end{tabular}

${ }^{\mathrm{a}} \mathrm{P}<0.01$ vs. control; ${ }^{\mathrm{b}} \mathrm{P}<0.01$ vs. Diabetes/Sham; ${ }^{\mathrm{c}} \mathrm{P}<0.01$ vs. MI. MI, myocardial infarction; LVSP, left ventricular systolic pressure; LVEDP, LV end-diastolic pressure.

Table II. CK-MB and cTnT levels in serum.

\begin{tabular}{lcr}
\hline Group & CK-MB $(\mathrm{ng} / \mathrm{ml})$ & \multicolumn{1}{c}{ cTnT (ng/l) } \\
\hline Control & $4.102 \pm 0.838$ & $49.778 \pm 13.641$ \\
Diabetes & $3.864 \pm 0.890$ & $56.656 \pm 11.491$ \\
Diabetes/Sham & $4.554 \pm 1.050$ & $55.806 \pm 15.380$ \\
MI & $7.337 \pm 1.455$ & $146.272 \pm 27.636$ \\
Diabetes/MI & $9.016 \pm 1.456^{\mathrm{a}}$ & $167.183 \pm 31.626^{\mathrm{a}}$
\end{tabular}

${ }^{\mathrm{a}} \mathrm{P}<0.01$ vs. Diabetes/Sham. CK-MB, creatinine kinase-MB; cTnT, cardiac troponin T; MI, myocardial infarction.

Statistical analysis. Statistical analyses for comparisons were performed by ANOVA followed by Tukey's post-hoc test using GraphPad Prism version 5.01 (GraphPad Software, Inc., San Diego, CA, USA). Data are expressed as mean \pm standard deviation. $\mathrm{P}<0.05$ was considered to indicate a statistically significant difference.

\section{Results}

Effects of diabetes and MI on ECG. As shown in Fig. 1, the rats in the Control group showed normal patterns of ECG. ST-segments were markedly elevated in rats in the Diabetes/MI group as compared with the Diabetes/Sham group or the MI group.

Diabetes aggravates heart dysfunction in rat hearts after MI. The LVEDP, LVSP and heart rate were recorded to assess heart function. As shown in Table I, diabetes increased LVEDP, while decreased LVSP and heart rate compared with the Control group. MI increased LVEDP and decreased LVSP in diabetic rats compared with the Sham-operated diabetic rats. The rats in the Diabetes/MI group exhibited elevation in LVEDP and reductions in LVSP and heart rate compared with the Diabetes/Sham group or MI group.

Diabetes aggravates myocardial injury in rat hearts after MI. To examine whether diabetes aggravates myocardial injury in rat hearts after MI, serum levels of CK-MB and cTnT were measured by ELISA. The results showed that STZ-induced diabetes had no significant effect on CK-MB and cTnT
(Table II). Serum CK-MB and cTnT levels were higher in the Diabetes/MI group than those in the Diabetes/Sham group or the MI group.

Diabetes enlarges myocardial infarct size in rats after MI. Myocardial infarct size was measured by TTC staining. As shown in Fig. 2, there was no MI in the rats of the Control, Diabetes or Diabetes/Sham group. The infarct sizes in diabetic rats after $\mathrm{MI}(43.614 \pm 9.615) \%$ were larger than those in both the Diabetes/Sham group (0\%) and the MI group $(32.430 \pm 6.890) \%$.

Diabetes induces pathological changes in rat hearts after MI. In the Control group, the myocardial cells were regularly arranged and did not show any pathological changes (Fig. 3A). The myocardial tissues of the Diabetes group, Diabetes/Sham group, MI group and Diabetes/MI group exhibited pathological changes in infarcted border zone, including disordered arrangement and rupture of myocardial fibers, nuclear condensation/fragmentation, myocardial interstitial edema, and inflammatory cell infiltration.

Diabetes promotes myocardial cell apoptosis in rat hearts after MI. Cell apoptosis was then evaluated by TUNEL assay. As shown in Fig. 3B, few apoptotic cells were observed in rat hearts from the Control group. TUNEL-positive cell rate in the Diabetes group was significantly increased as compared with that in the Control group. Diabetes complicated by MI further led to increased percentage of apoptotic cells compared with the Diabetes/Sham or MI group. Next, the levels of apoptosis-related proteins Bcl-2 and Bax were examined by Western blotting. The results showed that the Bcl-2 level was lower and Bax was higher in the Diabetes group than those in the Control group (Fig. 3C). MI further decreased Bcl-2 expression and increased Bax expression in diabetic rats compared with the Diabetes/Sham or MI group. Diabetes aggravated MI by inducing cell apoptosis.

Diabetes activates ER stress in rat hearts after MI. To investigate the effect of diabetes on ER stress in MI rats, GRP78 and CHOP levels in myocardial tissues were examined by western blotting. The results showed that STZ-induced diabetes elevated GRP78 and CHOP levels compared with the control rats (Fig. 4A). Additionally, STZ-induced diabetes further activated ER stress in MI rats, as evidenced by GRP78 and CHOP elevation. 

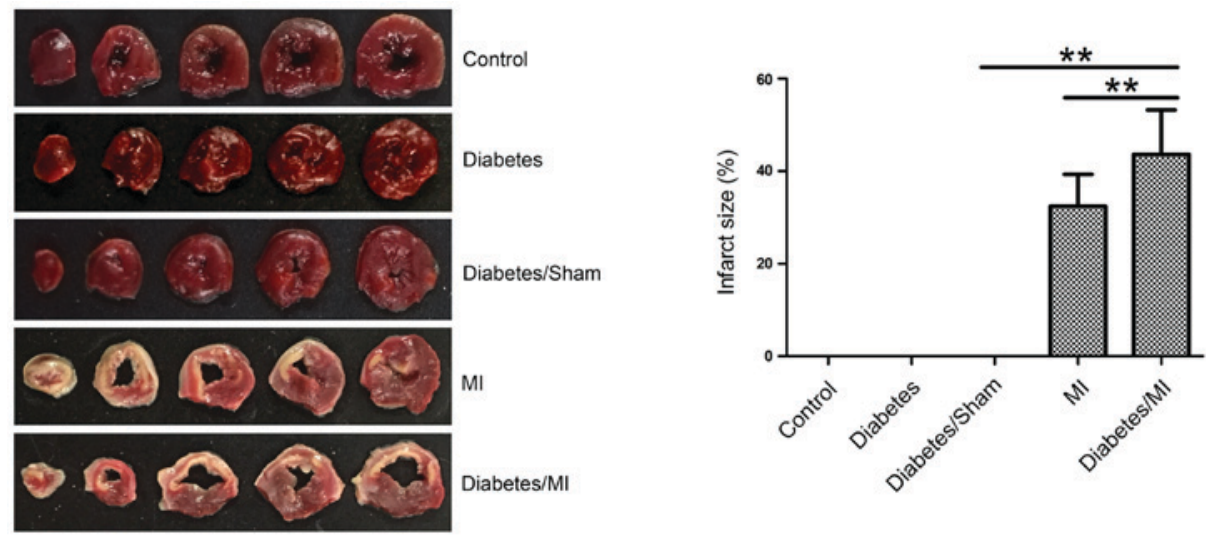

Figure 2. Infarct size by TTC staining. The rat hearts were stained with TTC and the percentage of infarct size was then calculated. Values are presented as the mean \pm standard deviation. ${ }^{* *} \mathrm{P}<0.01$, as indicated. TTC, triphenyltetrazolium chloride; MI, myocardial infarction.

A
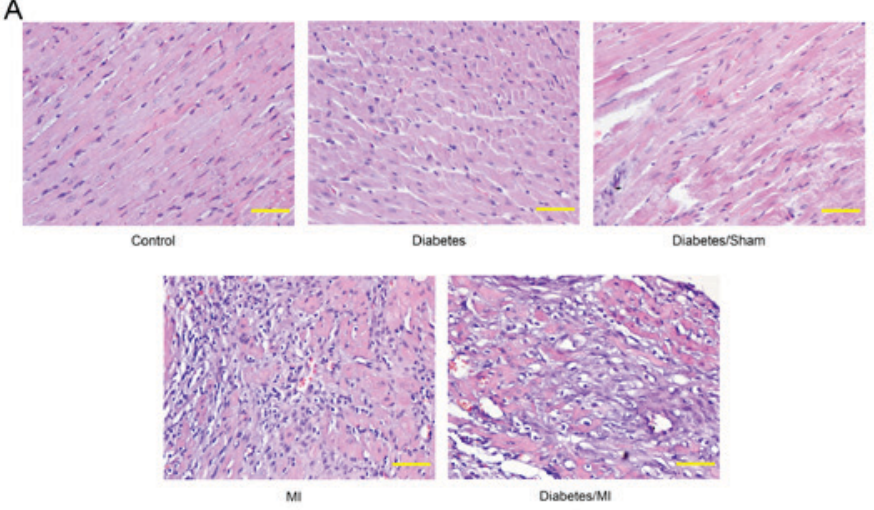

C

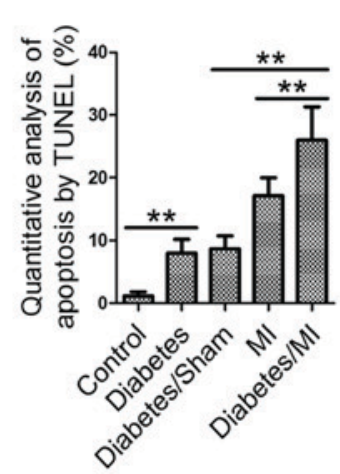

B
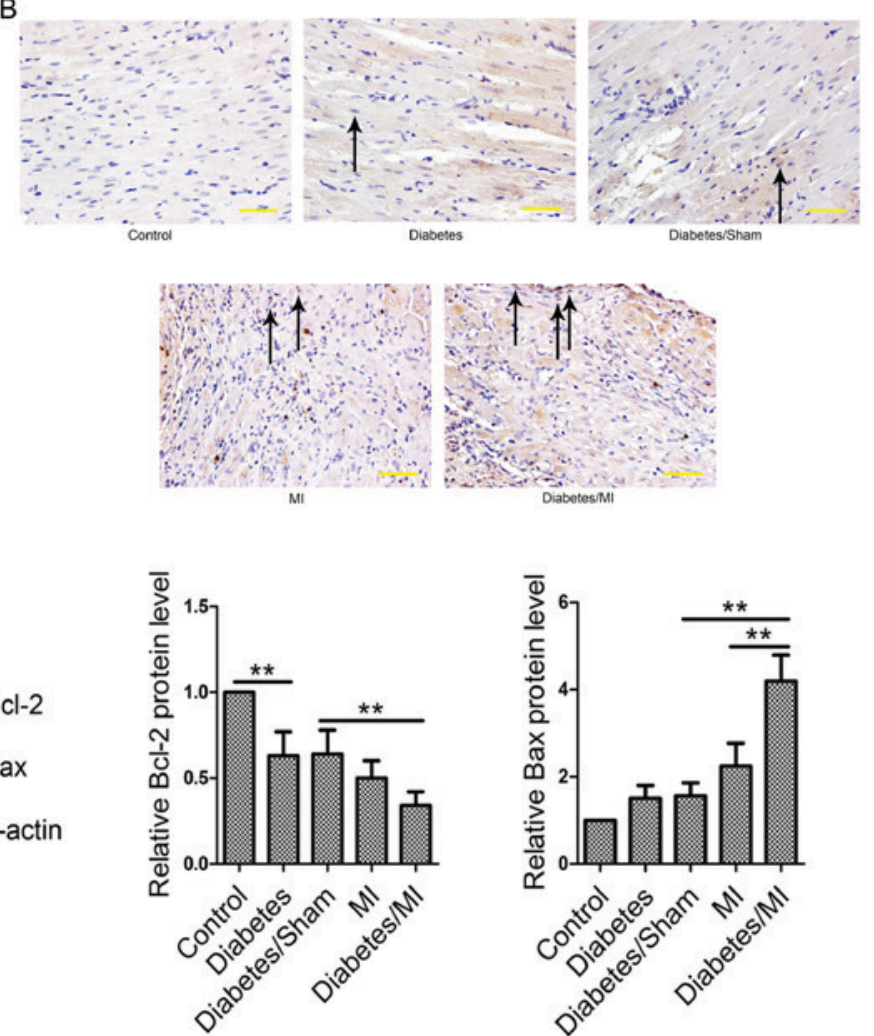

Figure 3. Pathological changes and cell apoptosis in myocardial tissues. (A) Hematoxylin and eosin staining was performed to evaluate the pathological changes in myocardial tissues. Scale bars=50 $\mu \mathrm{m}$. (B) TUNEL assay of infarcted border zone. Black arrows indicate apoptotic myocardial cells. Scale bars $=50 \mu \mathrm{m}$. (C) Analyses of Bcl-2 and Bax by western blotting. $\beta$-actin served as an internal control. Values are presented as the mean \pm standard deviation. ${ }^{* *} \mathrm{P}<0.01$, as indicated. TUNEL, terminal deoxynucleotidyl-transferase-mediated dUTP nick end labeling; Bcl-2, B-cell lymphoma 2; Bax, Bcl-2-associated X protein; MI, myocardial infarction.

Disruption of ER redox homeostasis in diabetic rats with MI. The results showed that diabetes upregulated Erol $\alpha$, Ero1 $\beta$ and PDI protein levels as compared with the Control group (Fig. 4B). The levels of Ero1 $\alpha$, Ero1 $\beta$ and PDI were greatly increased in diabetic rats with MI compared with the Diabetes/Sham group or MI group.

CHOP knockdown reverses the reduced cell viability and cell cycle arrest. Firstly, in our in vitro studies, CHOP expression was evaluated by western blotting post-siRNA transfection.
The results showed that CHOP siRNA transfection significantly reduced CHOP expression in $\mathrm{H} 9 \mathrm{c} 2$ cells (Fig. 5A). Cell viability was measured by CCK-8 assay. Treatment with HG or hypoxia resulted in significant decreases in cell viability as compared with the control cells (Fig. 5B). Additionally, $\mathrm{H} 9 \mathrm{c} 2$ cells exposed to both $\mathrm{HG}$ and hypoxic treatments showed lower cell viability than the single treatment group. However, siCHOP transfection partially restored the reduced cell viability induced by HG and hypoxia compared with the siNC-transfected cells. Then, cell cycle distribution was 
A
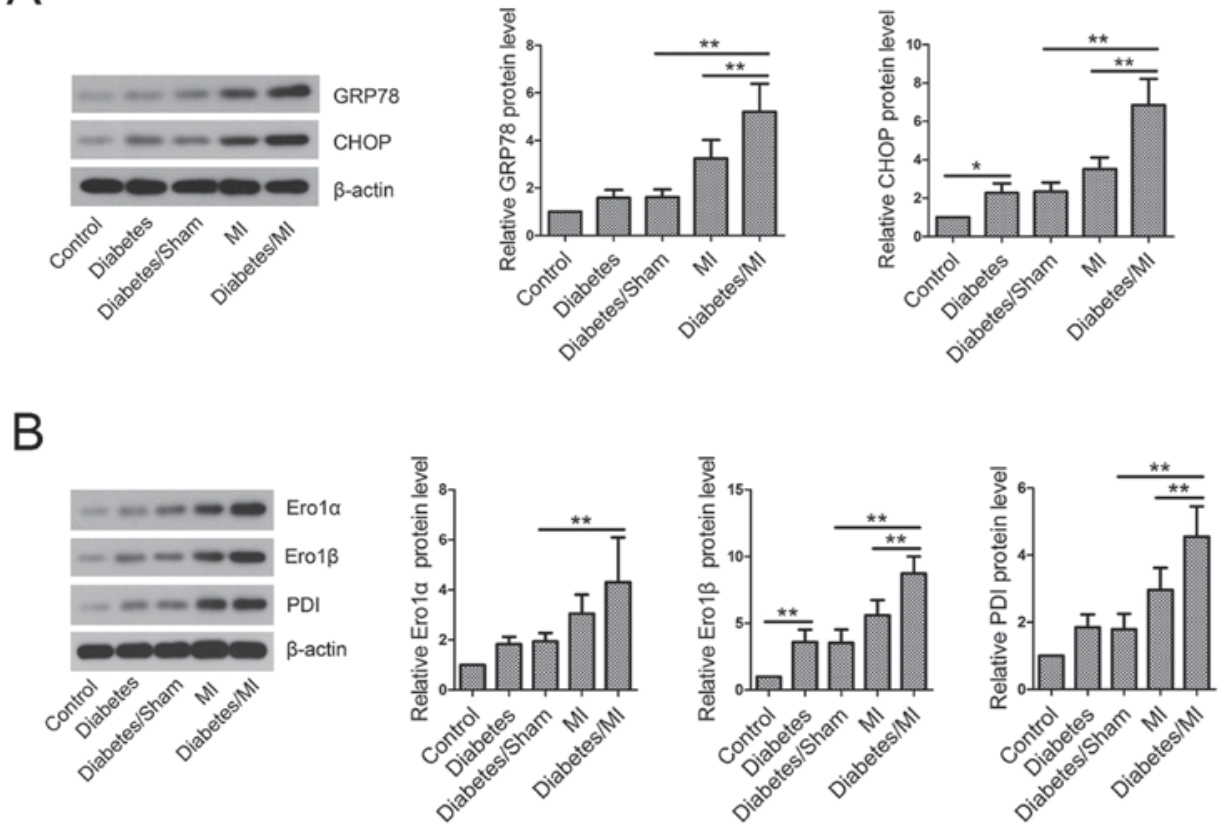

Figure 4. Endoplasmic reticulum stress and redox homeostasis in vivo. Total proteins were isolated from myocardial tissues and subjected to western blotting. $\beta$-actin served as an internal control. (A) Analyses of GRP78 and CHOP. (B) Analyses of Erol $\alpha$, Ero1 $\beta$ and PDI. Values are presented as the mean \pm standard deviation. ${ }^{*} \mathrm{P}<0.05$ and ${ }^{* *} \mathrm{P}<0.01$, as indicated. GRP78, glucose-regulated protein 78; CHOP, CCAAT/enhancer binding protein-homologous protein; Ero1, endoplasmic reticulum oxidoreductase 1; PDI, protein disulfide isomerase; MI, myocardial infarction.

A
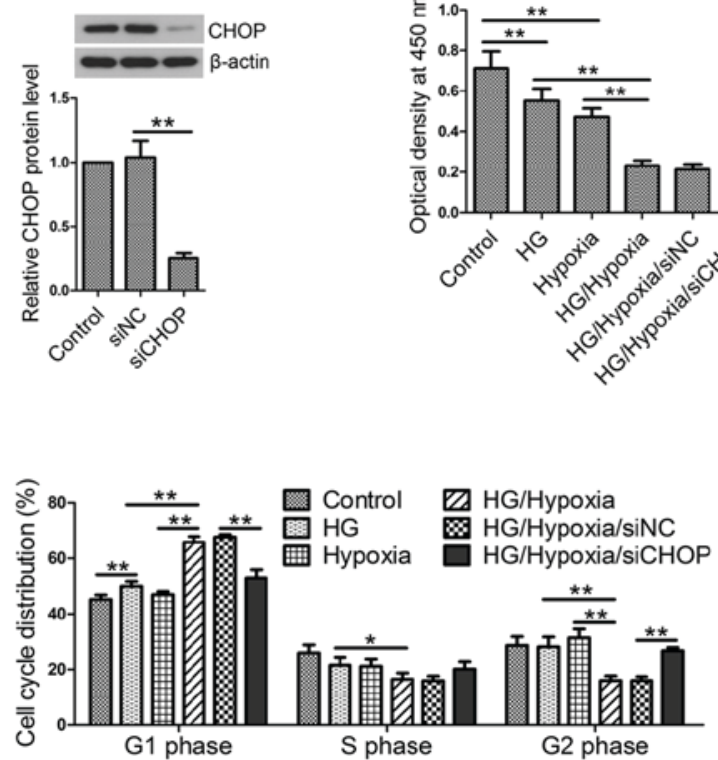

\section{B}

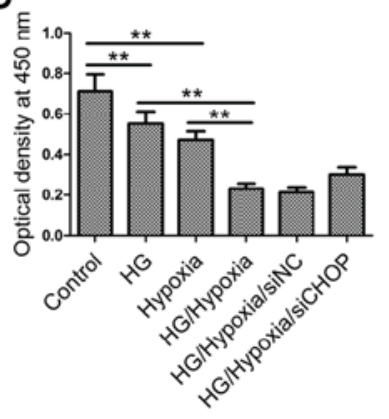

G2 phase
C
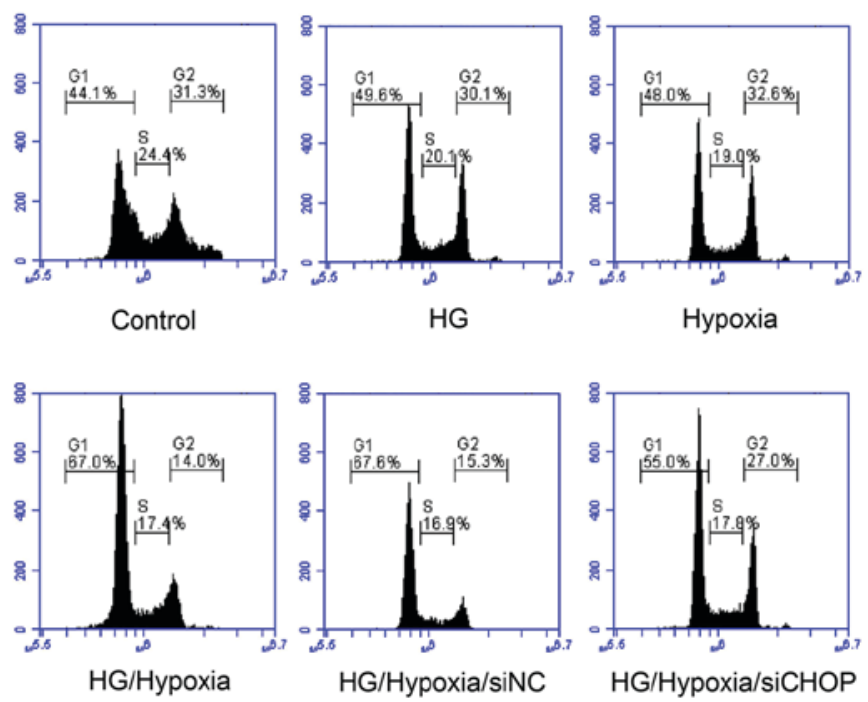

Figure 5. Cell proliferation and cell cycle progression in vitro. (A) CHOP protein levels were measured by western blotting post-siRNA transfection. (B) Cell Counting Kit- 8 assay. (C) Flow cytometric analysis of cell cycle progression. Values are presented as the mean \pm standard deviation. ${ }^{*} \mathrm{P}<0.05$ and ${ }^{* *} \mathrm{P}<0.01$, as indicated. CHOP, CCAAT/enhancer binding protein-homologous protein; HG, high glucose; NC, negative control; si/siRNA, small interfering RNA.

analyzed by flow cytometry. The results showed that HG followed by hypoxic treatment significantly arrested the cell cycle at the G1 phase, whereas CHOP knockdown reversed the cell cycle arrest (Fig. 5C).

CHOP knockdown reduces cell apoptosis rate in an in vitro model. Cell apoptosis was evaluated by Annexin V-FITC/PI assay and Hoechst staining. The results showed that exposure of $\mathrm{H} 9 \mathrm{c} 2$ cells to $\mathrm{HG}$ and/or hypoxia resulted in a remarkable increase in cell apoptotic rate (Fig. 6A and B) as well as upregulation of Bax and downregulation of Bcl-2 (Fig. 6C). However, this pro-apoptotic effect was partially abolished by CHOP knockdown.

CHOP knockdown inhibits ER stress and maintains ER redox homeostasis in $H 9 c 2$ cells. Then, the effects of $\mathrm{CHOP}$ 
A
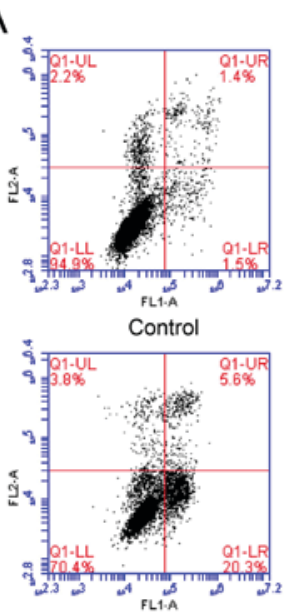

HG/Hypoxia

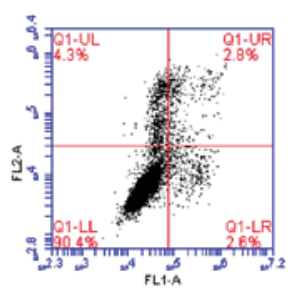

HG

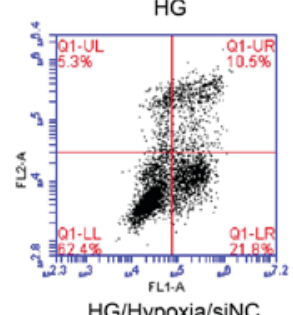

HG/Hypoxia/siNC

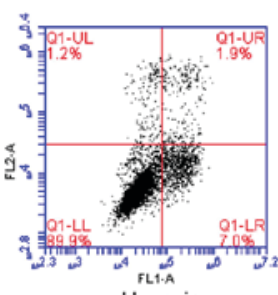

Hypoxia

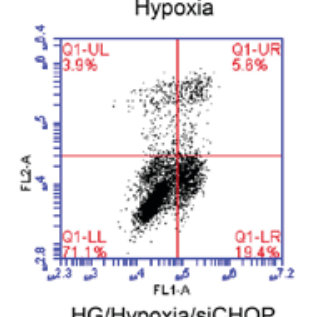

HG/Hypoxia/siCHOP

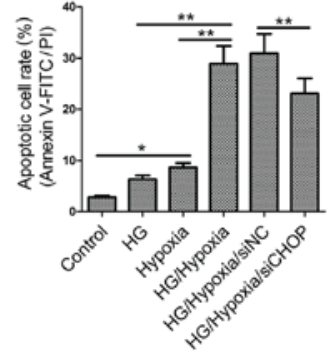

B

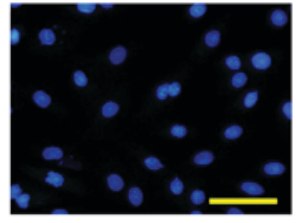

Control

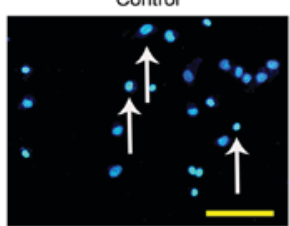

HG/Hypoxia
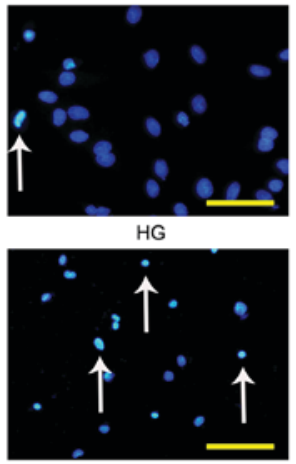

HG/Hypoxia/siNC

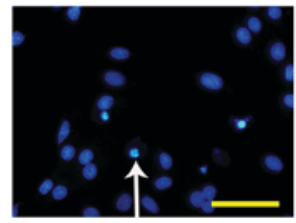

Hypoxia

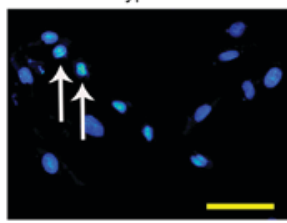

HG/Hypoxia/sicHOP

C
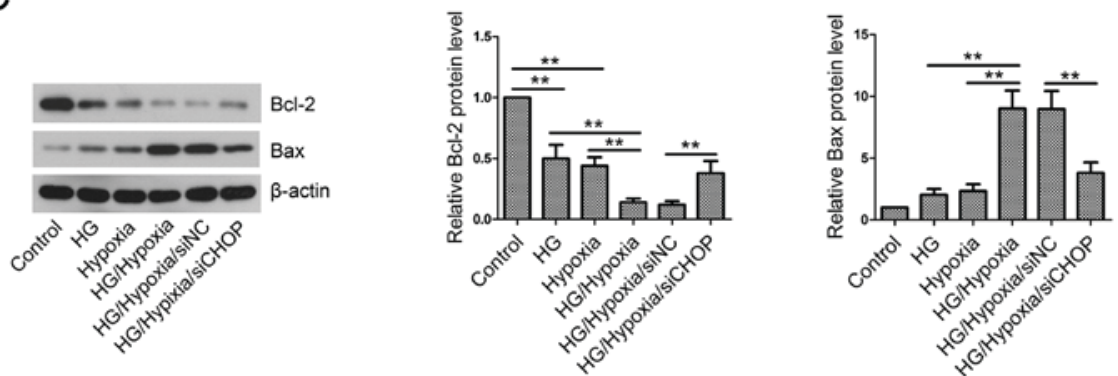

Figure 6. Myocardial cell apoptosis in vitro. (A) Myocardial cell apoptosis was examined by Annexin V-FITC/PI assay. (B) Hoechst staining. White arrows indicate apoptotic cells. Scale bars $=50 \mu \mathrm{m}$. (C) Western blotting analyses of Bcl-2 and Bax. $\beta$-actin served as an internal control. Values are presented as the mean \pm standard deviation. ${ }^{*} \mathrm{P}<0.05$ and ${ }^{* *} \mathrm{P}<0.01$, as indicated. Statistical analyses for comparisons were performed using ANOVA followed by Tukey's post-hoc test. CHOP, CCAAT/enhancer binding protein-homologous protein; HG, high glucose; NC, negative control; si/siRNA, small interfering RNA; Bcl-2, B-cell lymphoma 2; Bax, Bcl-2-associated X protein; FITC, fluorescein isothiocyanate; PI, propidium iodide.

knockdown on ER stress and ER redox homeostasis were investigated by western blotting. HG incubation followed by hypoxic treatment induced ER stress and resulted in an imbalance in ER redox homeostasis, as evidenced by upregulated levels of GRP78, CHOP, Erol $\alpha$, Ero1 $\beta$ and PDI (Fig. 7). However, CHOP knockdown partly restored the elevated levels of these key proteins.

\section{Discussion}

Clinical studies have revealed that CVD is the major cause of morbidity and mortality in diabetics (15). Patients with diabetes are more susceptible to I/R injury than non-diabetic patients (16). Therefore, effective therapeutic targets are urgently needed to alleviate MI in diabetes.

In the present study, diabetic SD rats induced by a single injection of STZ were subjected to LAD coronary artery ligation. Severity of myocardial injury is associated with the levels of serum enzymes, such as lactate dehydrogenase (LDH), CK-MB and cTnT (17). Moreover, cTnT is more specific and sensitive than CK-MB in the diagnosis of AMI (18). Zhang et al (19) have found that cardiac enzymes, including CK-MB and cTnT, are higher in the MI group 

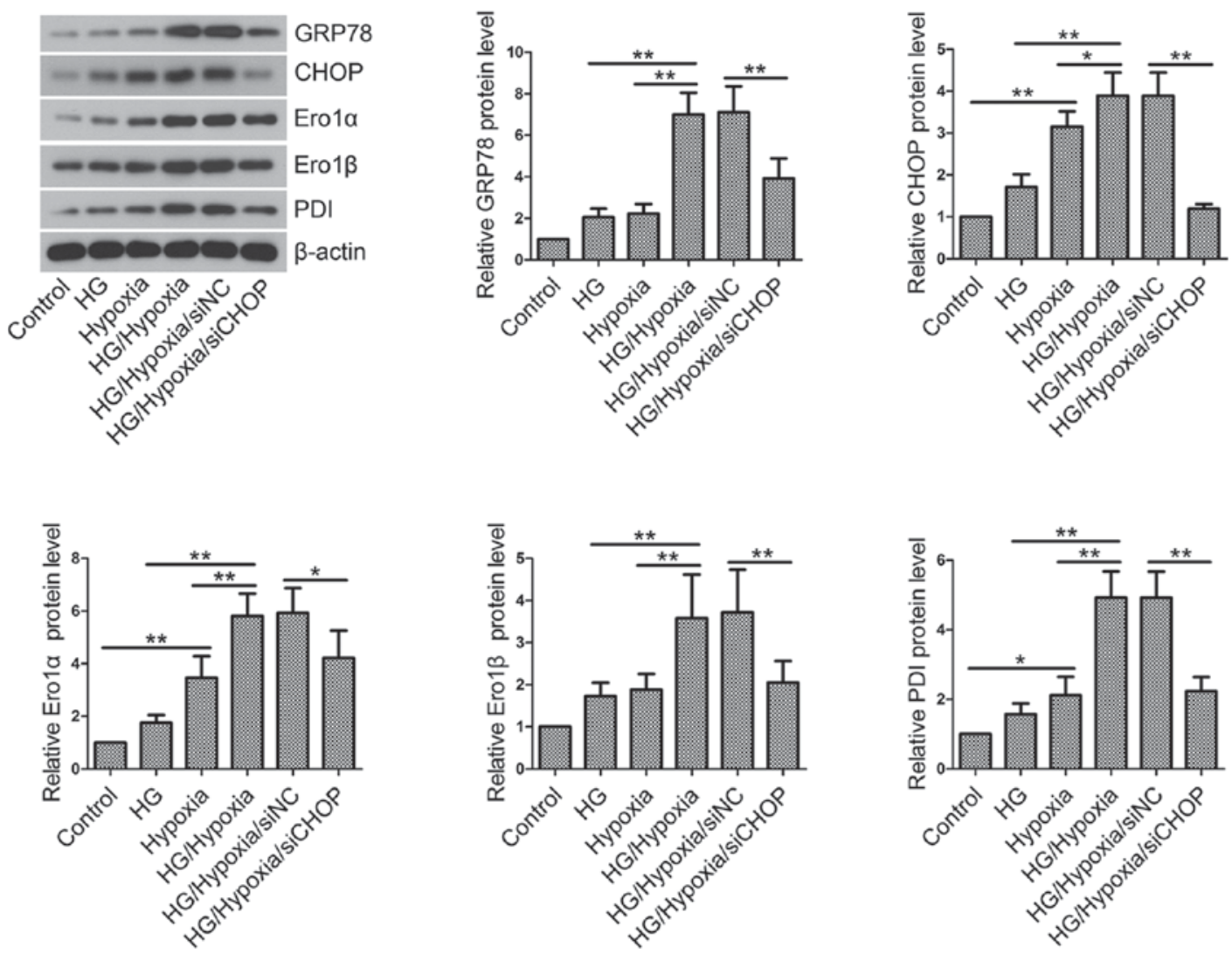

Figure 7. Endoplasmic reticulum stress and redox homeostasis in vitro. Total proteins were isolated from H9c2 cells in each group and western blotting was performed to measure GRP78, CHOP, Erol $\alpha$, Ero1 $\beta$ and PDI levels. $\beta$-actin served as an internal control. Values are presented as the mean \pm standard deviation. " $\mathrm{P}<0.05$ and ${ }^{* *} \mathrm{P}<0.01$, as indicated. GRP78, glucose-regulated protein 78; CHOP, CCAAT/enhancer binding protein-homologous protein; Ero1, endoplasmic reticulum oxidoreductase 1; PDI, protein disulfide isomerase; HG, high glucose; NC, negative control; si/siRNA, small interfering RNA.

than the Control group. Therefore, serum levels of CK-MB and $\mathrm{cTnT}$ were measured and the pathological changes were evaluated in diabetic rats following MI. The results showed that diabetes significantly increased CK-MB and cTnT levels and induced pathological changes in MI rats, which are consistent with previous findings (20). These data suggest that diabetes may aggravate MI-induced myocardial injury.

LVEDP and LVSP are indicators of heart function $(21,22)$. LVEDP reflects the cardiac diastolic function, while LVSP indicates the cardiac contractile function (23). In the present study, the results showed that LVEDP was increased and LVSP was decreased in STZ-induced diabetic rats with MI as compared with the Diabetes/Sham group or MI group, indicating that diabetes may impair heart function in rats with MI by altering hemodynamic parameters. The obtained results are in line with previous findings (24).

Previous evidences have revealed that myocardial apoptosis also plays a vital role in the progression of MI in humans and animal models (25). In the present study, myocardial apoptosis was investigated in diabetes complicated by MI in animal models. Myocardial I/R injury or MI can lead to myocardial apoptosis, which is correlated with infarct size and ventricular dysfunction (26). Infarct size is commonly used to evaluate the severity of I/R injury (27). The results showed that diabetes significantly increased infarct size and induced myocardial apoptosis in rats with MI, suggesting that diabetes may aggravate MI by enhancing infarct size enlargement and myocardial apoptosis.

GRP78 is a glucose-regulated protein and a major molecular chaperone in ER (28). GRP78 is a marker of ER stress (29). It has been reported that GRP78 is associated with folding of newly synthesized protein, translocation across the ER membrane and regulation of $\mathrm{Ca}^{2+}$ homeostasis (28). GRP78 forms a protein complex with PERK, IRE1 (inositol-requiring transmembrane kinase/endonuclease) and ATF6 under physiological conditions (30). When excessive misfolded proteins are accumulated in ER or under ER stress, these proteins are released from the protein complex and activated. Finally, the folding capacities of ER and CHOP expression are enhanced (30). CHOP is ubiquitously expressed, including normal myocardial tissues, at low levels in the cytosol under physiological conditions; while it accumulates in the nucleus when activated (31). Previous evidences have demonstrated that ER stress is associated with the development of cerebral and myocardial ischemia/reperfusion injury $(32,33)$. ER stress can lead to cell apoptosis (34). ER stress-induced apoptosis has been shown to be associated with transcription factors, caspase family proteins, Bcl-2 family members and JNK signaling pathway (35). CHOP is the first identified regulator that participates in ER stress-induced apoptosis (31). It has been reported that apoptosis is attenuated in CHOP-deficient mice in response to ER stress (36). In the present study, the results showed that the protein levels of GRP78 and CHOP 
were greatly higher in the Diabetes/MI group than those in the MI and Diabetes/Sham group. The results indicate that diabetes may aggravate MI by modulating ER stress and CHOP may be a key mediator in ER stress-induced apoptosis in an animal model of diabetes and MI.

Bcl-2 family members are involved in apoptosis, including pro-apoptotic protein Bax and anti-apoptotic protein Bcl-2 (37). CHOP overexpression results in a reduction of Bcl-2 and promotes Bax translocation to mitochondria, thereby regulating the mitochondrial apoptosis pathway; whereas, Bcl-2 overexpression reverses CHOP-induced apoptosis (38). The present study investigated whether $\mathrm{Bax}$ and $\mathrm{Bcl}-2$ participated in CHOP-induced apoptosis in diabetes complicated by MI. The results showed that the rats in the Diabetes/MI group exhibited higher Bax and lower Bcl-2 than those in the MI group or Diabetes/Sham group. These results suggest that Bax and $\mathrm{Bcl}-2$ may be the downstream regulators in CHOP-induced apoptosis in this animal model.

There are two isoforms of Ero1 in human cells, namely Erol $\alpha$ and Ero1 $\beta$. Erol $\alpha$ is identified in almost all cell types, while Ero1 $\beta$ is only expressed in select tissues (39). Erol $\alpha$ and Ero1 $\beta$ expression levels can be elevated by hypoxia and UPR (40). PDI (also named PDIA1) belongs to PDI family, which contains 21 family members. The family members have at least one thioredoxin-like $\beta \alpha \beta \alpha \beta \alpha \beta \beta \alpha$ domain $(41,42)$. Both Erol $\alpha$ and Ero1 $\beta$ can catalyze the reoxidation of PDI, in which oxygen was consumed to produce hydrogen peroxide (43). Previous evidence has revealed that the calcium signaling pathway is a mechanism of CHOP-induced apoptosis (44). Erol $\alpha$, a target gene of $\mathrm{CHOP}$, promotes ER calcium channel IP3R1-induced calcium release and thereby induces the activation of calcium-sensing enzyme CaMKII, eventually leading to apoptosis (44). In this study, Erol $\alpha$, Ero1 $\beta$ and PDI levels were examined by western blotting. The results showed that diabetes complicated with MI significantly elevated Erol $\alpha$, Ero1 $\beta$ and PDI levels compared with the MI group or Diabetes/Sham group. The results suggest that diabetes may exacerbate MI by disrupting ER redox homeostasis.

Consistent with previous reports $(45,46)$, our in vivo studies demonstrated that ER stress was activated in the development of ischemic injury in diabetes. Based on the in vivo findings in diabetic rats with $\mathrm{MI}$, an in vitro model was established using $\mathrm{H} 9 \mathrm{c} 2$ cells. In the present study, siCHOP-transfected H9c2 cells were incubated with $\mathrm{HG}$ and subjected to hypoxic treatment to investigate the role of CHOP.

Woo et al (47) have reported that delivery of cyclin A2 activates cell cycle in cardiomyocytes and improves heart function in a rat model of ischemic heart failure. Pasumarthi et al (48) have found that cyclin D2 overexpression promotes DNA synthesis in cardiomyocytes and infarct regression in transgenic mice. The aforementioned evidences suggest that cardiomyocyte proliferation is critical for maintaining heart function in injured heart. Thus, in the present study, cell proliferation was assessed by CCK-8 assay and flow cytometry. The results showed that HG combined with hypoxia reduced cell viability and caused cell cycle arrest at G1 phase. ER stress-induced apoptosis contributes to progression of acute MI and anti-apoptotic treatment can reduce infarct size in rats with MI (11). Our in vitro studies showed that HG combined with hypoxia induced cell apoptosis, activated ER stress and dysregulated ER redox homeostasis, which were consistent with our in vivo results. However, CHOP knockdown by specific siRNA transfection partly reversed the effect of $\mathrm{HG}$ and hypoxia.

In summary, CHOP knockdown alleviated MI in diabetes via the GRP78/CHOP and Ero1/PDI signaling pathways. $\mathrm{CHOP}$ is expected to be a promising target for the treatment of diabetes complicated by MI.

\section{References}

1. Bai Y, Wang X, Zhao S, Ma C, Cui J and Zheng Y: Sulforaphane protects against cardiovascular disease via Nrf2 activation. Oxid Med Cell Longev 2015: 407580, 2015.

2. Yang ZH, Emma-Okon B and Remaley AT: Dietary marine-derived long-chain monounsaturated fatty acids and cardiovascular disease risk: A mini review. Lipids Health Dis 15: 201, 2016.

3. Bernardi S, Michelli A,Zuolo G, Candido R and Fabris B: Update on RAAS modulation for the treatment of diabetic cardiovascular disease. J Diabetes Res 2016: 8917578, 2016.

4. Lipes MA and Galderisi A: Cardiac autoimmunity as a novel biomarker, mediator and therapeutic target of heart disease in type 1 diabetes. Curr Diab Rep 15: 30, 2015.

5. Gregg EW, Cheng YJ, Saydah S, Cowie C, Garfield S, Geiss L and Barker L: Trends in death rates among U.S. adults with and without diabetes between 1997 and 2006: Findings from the National Health Interview Survey. Diabetes Care 35: 1252-1257, 2012.

6. Lee JM: Nuclear receptors resolve endoplasmic reticulum stress to improve hepatic insulin resistance. Diabetes Metab J 41: 10-19, 2017.

7. Phillips MJ and Voeltz GK: Structure and function of ER membrane contact sites with other organelles. Nat Rev Mol Cell Biol 17: 69-82, 2016.

8. Chen Y, Tsai YH and Tseng SH: HDAC inhibitors and RECK modulate endoplasmic reticulum stress in tumor cells. Int J Mol Sci 18: pii: E258, 2017.

9. Lee YT, Lin HY, Chan YW, Li KH, To OT, Yan BP, Liu T, Li G, Wong WT, Keung W and Tse G: Mouse models of atherosclerosis: A historical perspective and recent advances. Lipids Health Dis 16: 12, 2017.

10. Oyadomari S, Koizumi A, Takeda K, Gotoh T, Akira S, Araki E and Mori M: Targeted disruption of the Chop gene delays endoplasmic reticulum stress-mediated diabetes. J Clin Invest 109: 525-532, 2002.

11. Shi ZY, Liu Y, Dong L, Zhang B, Zhao M, Liu WX, Zhang X and Yin XH: Cortistatin improves cardiac function after acute myocardial infarction in rats by suppressing myocardial apoptosis and endoplasmic reticulum stress. J Cardiovasc Pharmacol Ther: pii: $1074248416644988,2016$.

12. Rao J, Zhang C, Wang P, Lu L, Qian X, Qin J, Pan X, Li G, Wang $X$ and Zhang F: C/EBP homologous protein (CHOP) contributes to hepatocyte death via the promotion of ERO1a signalling in acute liver failure. Biochem J 466: 369-378, 2015.

13. Takada A, Miki T, Kuno A, Kouzu H, Sunaga D, Itoh T, Tanno M, Yano T, Sato T, Ishikawa S and Miura T: Role of ER stress in ventricular contractile dysfunction in type 2 diabetes. PLoS One 7: e39893, 2012.

14. Barr LA, Shimizu Y, Lambert JP, Nicholson CK and Calvert JW: Hydrogen sulfide attenuates high fat diet-induced cardiac dysfunction via the suppression of endoplasmic reticulum stress. Nitric Oxide 46: 145-156, 2015.

15. Rawal S, Manning P and Katare R: Cardiovascular microRNAs: As modulators and diagnostic biomarkers of diabetic heart disease. Cardiovasc Diabetol 13: 44, 2014.

16. Kuusisto J and Laakso M: Update on type 2 diabetes as a cardiovascular disease risk equivalent. Curr Cardiol Rep 15: 331, 2013.

17. Lu SF, Huang Y, Wang N, Shen WX, Fu SP, Li Q, Yu ML, Liu WX, Chen X, Jing XY and Zhu BM: Cardioprotective effect of electroacupuncture pretreatment on myocardial ischemia/reperfusion injury via antiapoptotic signaling. Evid Based Complement Alternat Med 2016: 4609784, 2016.

18. Tiwari RP, Jain A, Khan Z, Kohli V, Bharmal RN, Kartikeyan S and Bisen PS: Cardiac troponins I and T: Molecular markers for early diagnosis, prognosis and accurate triaging of patients with acute myocardial infarction. Mol Diagn Ther 16: 371-381, 2012. 
19. Zhang J, Yu P, Chen M, Peng Q, Wang Z and Dong N: Remote ischaemic preconditioning and sevoflurane postconditioning synergistically protect rats from myocardial injury induced by ischemia and reperfusion partly via inhibition TLR4/MyD88/NF- $\kappa$ B signaling pathway. Cell Physiol Biochem 41: 22-32, 2017.

20. Ding M, Lei J, Han H, Li W, Qu Y, Fu E, Fu F and Wang X: SIRT1 protects against myocardial ischemia-reperfusion injury via activating eNOS in diabetic rats. Cardiovasc Diabetol 14: 143, 2015.

21. Liu J, Wang N, Xie F, Sun LH, Chen QX, Ye JH, Cai BZ, Yang BF and Ai J: Blockage of peripheral NPY Y1 and Y2 receptors modulates barorefex sensitivity of diabetic rats. Cell Physiol Biochem 31: 421-431, 2013.

22. Chang R, Li Y, Yang X, Yue Y, Dou L, Wang Y, Zhang W and Li X: Protective role of deoxyschizandrin and schisantherin A against myocardial ischemia-reperfusion injury in rats. PLoS One 8: e61590, 2013.

23. Xie F, Sun L, Su X, Wang Y, Liu J, Zhang R, Wang N, Zhao J, Ban T, Niu H and Ai J: Neuropeptide Y reverses chronic stress-induced baroreflex hypersensitivity in rats. Cell Physiol Biochem 29: 463-474, 2012.

24. Agrawal YO, Sharma PK, Shrivastava B, Arya DS and Goyal SN Hesperidin blunts streptozotocin-isoproternol induced myocardial toxicity in rats by altering of PPAR- $\gamma$ receptor. Chem Biol Interact 219: 211-220, 2014

25. Bussani R, Abbate A, Biondi-Zoccai GG, Dobrina A, Leone AM, Camilot D, Di Marino MP, Baldi F, Silvestri F, Biasucci LM and Baldi A: Right ventricular dilatation after left ventricular acute myocardial infarction is predictive of extremely high peri-infarctual apoptosis at postmortem examination in humans. J Clin Pathol 56: 672-676, 2003.

26. Wang X, Wang Q, Guo W and Zhu YZ: Hydrogen sulfide attenuates cardiac dysfunction in a rat model of heart failure: A mechanism through cardiac mitochondrial protection. Biosci Rep 31: 87-98, 2011.

27. Han Q, Zhang HY,Zhong BL, Zhang B and Chen H: Antiapoptotic effect of recombinant HMGB1 A-box protein via regulation of microRNA-21 in myocardial ischemia-reperfusion injury model in rats. DNA Cell Biol 35: 192-202, 2016.

28. Li J, Ni M, Lee B, Barron E, Hinton DR and Lee AS: The unfolded protein response regulator GRP78/BiP is required for endoplasmic reticulum integrity and stress-induced autophagy in mammalian cells. Cell Death Differ 15: 1460-1471, 2008.

29. Yu H, Zhen J, Yang Y, Gu J, Wu S and Liu Q: Ginsenoside Rg1 ameliorates diabetic cardiomyopathy by inhibiting endoplasmic reticulum stress-induced apoptosis in a streptozotocin-induced diabetes rat model. J Cell Mol Med 20: 623-631, 2016.

30. Bennett HL, Fleming JT, O'Prey J, Ryan KM and Leung HY: Androgens modulate autophagy and cell death via regulation of the endoplasmic reticulum chaperone glucose-regulated protein 78/BiP in prostate cancer cells. Cell Death Dis 1: e72, 2010.

31. Oyadomari S and Mori M: Roles of CHOP/GADD153 in endoplasmic reticulum stress. Cell Death Differ 11: 381-389, 2004.

32. DeGracia DJ and Montie HL: Cerebral ischemia and the unfolded protein response. J Neurochem 91: 1-8, 2004.

33. Zhang M, Yu LM, Zhao H, Zhou XX, Yang Q, Song F, Yan L, Zhai ME, Li BY, Zhang B, et al: 2,3,5,4'-Tetrahydroxystilbe ne-2-O- $\beta$-D-glucoside protects murine hearts against ischemia/reperfusion injury by activating Notch1/Hes1 signaling and attenuating endoplasmic reticulum stress. Acta Pharmaco Sin 38: 317-330, 2017.

34. Nakagawa T, Zhu H, Morishima N, Li E, Xu J, Yankner BA and Yuan J: Caspase-12 mediates endoplasmic-reticulum-specific apoptosis and cytotoxicity by amyloid-beta. Nature 403: 98-103, 2000 .
35. Kadowaki H, Nishitoh $\mathrm{H}$ and Ichijo H: Survival and apoptosis signals in ER stress: The role of protein kinases. J Chem Neuroanat 28: 93-100, 2004.

36. Zinszner H, Kuroda M, Wang X, Batchvarova N, Lightfoot RT, Remotti H, Stevens JL and Ron D: CHOP is implicated in programmed cell death in response to impaired function of the endoplasmic reticulum. Genes Dev 12: 982-995, 1998.

37. Gross A: BCL-2 family proteins as regulators of mitochondria metabolism. Biochim Biophys Acta 1857: 1243-1246, 2016.

38. Fu HY, Okada K, Liao Y, Tsukamoto O, Isomura T, Asai M, Sawada T, Okuda K, Asano Y, Sanada S, et al: Ablation of C/EBP homologous protein attenuates endoplasmic reticulum-mediated apoptosis and cardiac dysfunction induced by pressure overload. Circulation 122: 361-369, 2010.

39. Inaba K, Masui S, Iida H, Vavassori S, Sitia R and Suzuki M: Crystal structures of human Erolalpha reveal the mechanisms of regulated and targeted oxidation of PDI. EMBO J 29: 3330-3343, 2010.

40. Dias-Gunasekara S, Gubbens J, van Lith M, Dunne C, Williams JA, Kataky R, Scoones D, Lapthorn A, Bulleid NJ, Benham AM, et al: Tissue-specific expression and dimerization of the endoplasmic reticulum oxidoreductase Erolbeta. J Biol Chem 280: 33066-33075, 2005.

41. Kozlov G, Määttänen P, Thomas DY and Gehring K: A structural overview of the PDI family of proteins. FEBS J 277: 3924-3936, 2010.

42. Andreu CI, Woehlbier U, Torres M and Hetz C: Protein disulfide isomerases in neurodegeneration: From disease mechanisms to biomedical applications. FEBS Lett 586: 2826-2834, 2012.

43. Swiatkowska M, Padula G, Michalec L, Stasiak M, Skurzynski S and Cierniewski CS: Erolalpha is expressed on blood platelets in association with protein-disulfide isomerase and contributes to redox-controlled remodeling of alphaIIbbeta3. J Biol Chem 285: 29874-29883, 2010.

44. Scull CM and Tabas I: Mechanisms of ER stress-induced apoptosis in atherosclerosis. Arterioscler Thromb Vasc Biol 31: 2792-2797, 2011.

45. Su D,MaJ, Yang J,Kang Y,Lv Mand Li Y: Monosialotetrahexosy-1 ganglioside attenuates diabetes-associated cerebral ischemia/reperfusion injury through suppression of the endoplasmic reticulum stress-induced apoptosis. J Clin Neurosci 41: 54-59, 2017.

46. Yu L, Li S, Tang X, Li Z, Zhang J, Xue X, Han J, Liu Y, Zhang Y, Zhang Y, et al: Diallyl trisulfide ameliorates myocardial ischemia-reperfusion injury by reducing oxidative stress and endoplasmic reticulum stress-mediated apoptosis in type 1 diabetic rats: Role of SIRT1 activation. Apoptosis 22: 942-954, 2017.

47. Woo YJ, Panlilio CM, Cheng RK, Liao GP, Atluri P, Hsu VM, Cohen JE and Chaudhry HW: Therapeutic delivery of cyclin A2 induces myocardial regeneration and enhances cardiac function in ischemic heart failure. Circulation 114 (1 Suppl): I206-I213, 2006.

48. Pasumarthi KB, Nakajima H, Nakajima HO, Soonpaa MH and Field LJ: Targeted expression of cyclin D2 results in cardiomyocyte DNA synthesis and infarct regression in transgenic mice. Circ Res 96: 110-118, 2005.

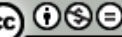

This work is licensed under a Creative Commons Attribution-NonCommercial-NoDerivatives 4.0 International (CC BY-NC-ND 4.0) License. 